\title{
Grain-structure development in heavily cold-rolled alpha-titanium
}

\author{
G.S. Dyakonov ${ }^{\text {a }}$, S. Mironov ${ }^{\text {b,*, S.V. Zherebtsov }}{ }^{\text {c }}$, S.P. Malysheva ${ }^{\text {d }}$, G.A. Salishchev ${ }^{\text {, }}$, \\ A.A. Salem ${ }^{\mathrm{e}, \mathrm{f}}$, S.L. Semiatin ${ }^{\mathrm{f}}$ \\ a Institute of Physics of Advanced Materials, Ufa State Aviation Technical University, 12 K. Marx Street, 450000 Ufa, Russia \\ ${ }^{\mathrm{b}}$ Department of Materials Processing, Graduate School of Engineering, Tohoku University, 6-6-02 Aramaki-aza-Aoba, Sendai 980-8579, Japan \\ ${ }^{\mathrm{c}}$ Laboratory of Bulk Nanostructured Materials, Belgorod State University, Pobeda 85, Belgorod 308015, Russia \\ ${ }^{\mathrm{d}}$ Institute for Metals Superplasticity Problems, Russian Academy of Science, 39 Khalturin Str., Ufa 450001, Russia \\ e Materials Resources LLC, Dayton, OH 45402, USA \\ ${ }^{\mathrm{f}}$ Air Force Research Laboratory, Materials and Manufacturing Directorate, AFRL/RXCM, Wright-Patterson AFB, OH 45433-7817, USA
}

\section{A R T I C L E I N F O}

\section{Article history:}

Received 4 February 2014

Received in revised form

27 March 2014

Accepted 27 March 2014

Available online 5 April 2014

Keywords:

EBSD

Nanostructured materials

Titanium alloys

Bulk deformation

Grain refinement

\begin{abstract}
A B S T R A C T
High-resolution electron back-scatter diffraction (EBSD) analysis was employed to establish mircostructure evolution in heavily cold-rolled alpha-titanium. After thickness reductions of 75\% to 96\%, significant microstructure and texture changes were documented. The surface area of high-angle grain boundaries was almost tripled, thus giving rise to an ultra-fine microstructure with a mean grain size of $0.6 \mu \mathrm{m}$. Moreover, orientation spread around typical 'split-basal' rolling texture substantially increased. These effects were suggested to be related to the enhancement of pyramidal $\langle c+a\rangle$ slip.
\end{abstract}

(c) 2014 Elsevier B.V. All rights reserved.

\section{Introduction}

The mechanical properties of metallic materials may be improved considerably by microstructure refinement. To attain a very fine structure with a grain size less than one micrometer, a variety of techniques based on severe deformation at low temperatures, such as high-pressure torsion, equal-channel angular extrusion, and 'abc' forging, are commonly used [1]. For commercial-purity titanium, it has been found, however, that the refinement imparted by these techniques is comparable to that produced by conventional cold rolling [2]. Such a finding has spurred a renewed interest in microstructure evolution during cold rolling of this material.

Microstructure evolution during rolling to relatively low thickness reductions ( $\leq 40 \%$ ) has been shown to be markedly influenced by mechanical twinning [3-8]. Extensive development of both compressive $\{11 \overline{2} 2\}\langle 11 \overline{2} \overline{3}\rangle$ and tensile $\{10 \overline{1} 2\}\langle 10 \overline{1} \overline{1}\rangle$ twins during rolling leads to rapid grain refinement [3-7]. On the other hand, grains favorably oriented for prism slip tend to remain untwinned $[4,6]$. This behavior gives rise to a bimodal grain structure consisting of finegrained (twinned) and coarse-grained (untwinned) areas [6].

\footnotetext{
* Corresponding author. Tel.: +8122 795 7353; fax: +8122795 7352 .

E-mail address: smironov@material.tohoku.ac.jp (S. Mironov).
}

At relatively large thickness reductions ( $\geq 40 \%$ ), grain refinement suppresses twinning activity, and slip becomes the dominant deformation mode $[9,10]$. Microstructure formation at these large strains has been hypothesized to be largely controlled by the development of deformation-induced dislocation boundaries $[5,8]$, but the details of this process are not clear. The objective of the present investigation, therefore, was to develop an understanding of microstructure evolution during cold-rolling of alpha titanium to large strains.

\section{Material and procedures}

The material used in the present investigation was commercialpurity titanium whose nominal chemical composition is given in Table 1 . The as-received material was pre-conditioned by severe 'abc' forging at temperatures in the range of $600-400{ }^{\circ} \mathrm{C}$ followed by annealing at $800{ }^{\circ} \mathrm{C}$ for $1 \mathrm{~h}$. This produced a fully recrystallized structure with a mean grain size of $\sim 35 \mu \mathrm{m}$, a large fraction (85\%) of high-angle boundaries, and a moderate crystallographic texture with (0002) basal planes inclined by $\sim 45^{\circ}$ to a rolling plane (supplementary data, Fig. S1).

Rolling samples measuring 30 (length) $\times 10$ (width) $\times 3$ (thickness) $\mathrm{mm}^{3}$ were machined from the recrystallized material. They were rolled at ambient temperature using a thickness reduction 
per pass of $10 \%$. to an overall total reduction of $25 \%, 50 \%, 75 \%$, or $96 \%$. using a rolling speed of $30 \mathrm{~mm} / \mathrm{s}$ in a cluster mill with $65-\mathrm{mm}$ diameter work rolls. To maintain consistency with the scientific literature, the typical flat-rolling convention was adopted in this work; i.e., the rolling, long-transverse, and thickness/normal directions were denoted as RD, TD, and ND, respectively.

Microstructure characterization was performed primarily via electron backscatter diffraction (EBSD) examination of the midthickness rolling plane (containing the RD and TD). For this purpose, samples were prepared using conventional metallographic techniques followed by long-term ( $24 \mathrm{~h})$ vibratory polishing with a colloidal-silica suspension. EBSD analysis was conducted with a JSM-7800F field-emission gun, scanning electron microscope (FEG-SEM) equipped with a TSL OIM ${ }^{\mathrm{TM}}$ EBSD system. To examine microstructure and texture evolution at different scales, several EBSD maps were acquired for each material condition (Table 2). For each diffraction pattern, nine Kikuchi bands were used to index the orientation thus minimizing the possibility of errors. The average confidence index (CI) for EBSD maps ranged from 0.2 to 0.64 (Table 2). By comparison, experiments on face-

Table 1

Nominal chemical composition (wt\%) of program material.

\begin{tabular}{lllllllll}
\hline Al & Fe & O & Si & C & N & H & other & Ti \\
\hline 0.30 & 0.30 & 0.20 & 0.10 & 0.07 & 0.04 & 0.01 & 0.30 & Bal. \\
\hline
\end{tabular}

Table 2

EBSD measurements.

\begin{tabular}{llllr}
\hline $\begin{array}{l}\text { Material } \\
\text { condition }\end{array}$ & $\begin{array}{l}\text { Scan step } \\
\text { size }(\mu \mathrm{m})\end{array}$ & $\begin{array}{l}\text { Map area } \\
\left(\mathrm{mm}^{2}\right)\end{array}$ & $\begin{array}{l}\text { Average } \\
\text { confidence index }\end{array}$ & $\begin{array}{l}\text { Number of } \\
\text { grains }\end{array}$ \\
\hline Unrolled material & 5.0 & 64.40 & 0.55 & 52,689 \\
& 0.1 & 0.08 & 0.64 & 243 \\
& & & & 29,060 \\
25\% reduction & 1.0 & 6.00 & 0.56 & 7970 \\
& 0.1 & 0.09 & 0.56 & 65,304 \\
& & & & 16,052 \\
50\% reduction & 0.5 & 1.00 & 0.41 & 130,940 \\
& 0.1 & 0.09 & 0.45 & 7299 \\
$75 \%$ reduction & 0.5 & 1.03 & 0.18 & 145,839 \\
& 0.1 & 0.02 & 0.32 & 17,317 \\
\hline
\end{tabular}

a

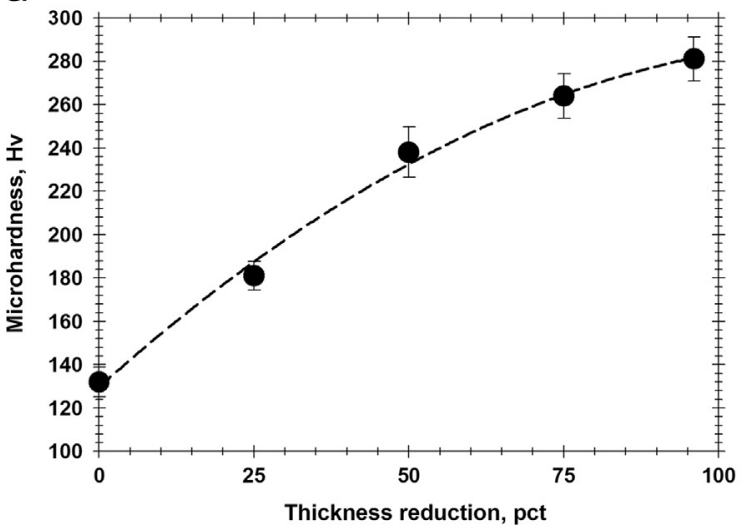

centered cubic materials have shown that the fraction of correctly indexed patterns with CIs greater than 0.1 is 95\% [11]. Nonindexed data points as well as points with low $\mathrm{CI}(\leq 0.1)$ were usually associated with grain-boundary regions. Grains comprising 3 or fewer pixels were automatically cleaned in the EBSD maps using the grain-dilation option in the TSL software. In addition, to eliminate spurious boundaries caused by software limitations, a lower-limit boundary misorientation cut-off of $2^{\circ}$ was used.

A $15^{\circ}$ criterion was used to differentiate low-angle boundaries (LABs) and high-angle boundaries (HABs). Because the microstructures developed at large strains are frequently characterized by a complex mixture of HABs and LABs, there is often confusion in the definition of grains. To clarify this issue, the term 'grain' in the present work was applied to denote a crystallite bordered by a continuous $\mathrm{HAB}$ perimeter. In all cases, the grain size was quantified by the determination of the area of each grain and the calculation of its circle-equivalent diameter, i.e., the so-called grain-reconstruction method [12].

To obtain an additional insight into microstructure evolution and material flow, the Vicker's microhardness was measured using a load of $500 \mathrm{~g}$ for $10 \mathrm{~s}$. At least 25 measurements were made in each case to obtain an average value.

\section{Results}

\subsection{Microhardness}

The influence of rolling strain on microhardness is illustrated in Fig. 1a. It is seen that the hardness doubled after rolling to $96 \%$ thickness reduction. On the other hand, the approximate hardening rate $\delta \mathrm{Hv} / \delta \varepsilon$ (where Hv is microhardness and $\varepsilon$ is true strain) was found to drop rapidly after a true strain of $50 \%$ (Fig. 1b). This effect may be related to the suppression of twinning at large strains [13] as well as with texture changes, discussed below. Furthermore, the strain hardening rate approached almost zero at the maximum level of deformation (Fig. 1b). This behavior may promote instability of material flow and the formation of deformation/shear bands.

\subsection{Structure morphology and grain size}

Selected portions of low- and high-resolution EBSD maps illustrating the grain structures which developed during rolling to different thickness reductions are summarized in Figs. 2-4. In the orientation maps shown in Figs. 2 and 3b, grains are colored according to the orientation of the local sample normal direction relative to the crystal coordinate system. In the grain-boundary

b

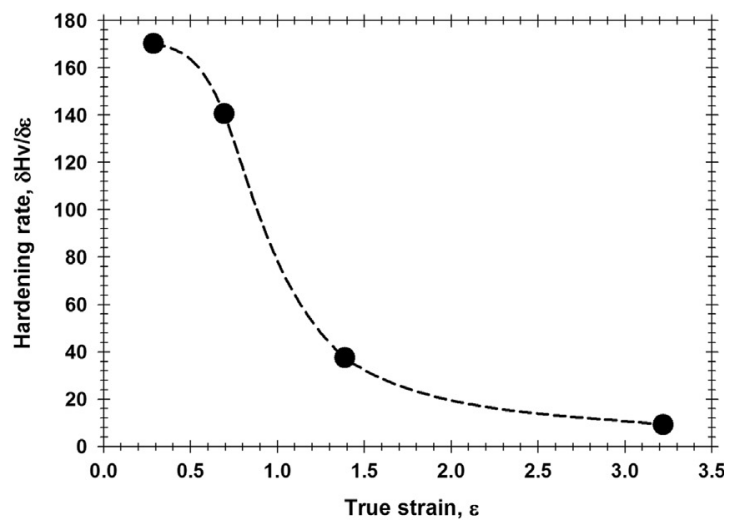

Fig. 1. Effect of rolling strain on microhardness (a) and hardening rate (b). In (a), error bars show standard deviation. 

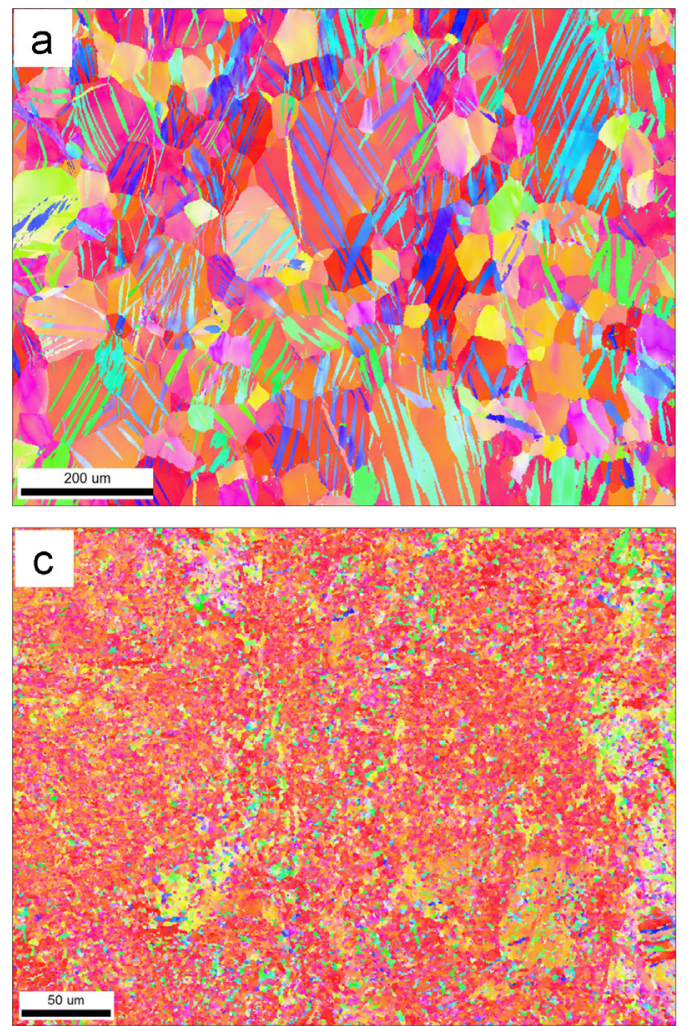
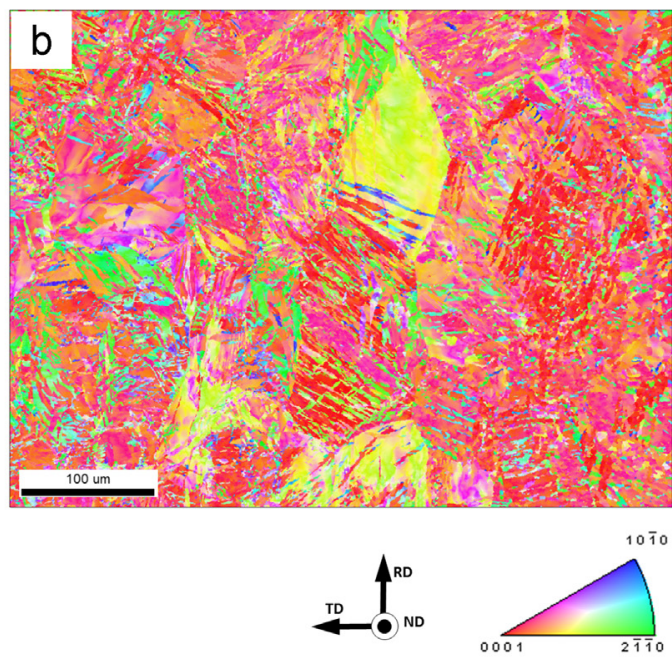

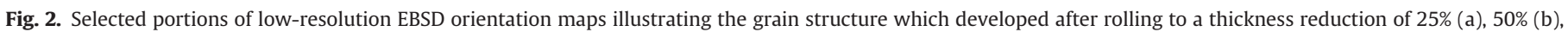
and $75 \%$ (c). In the maps, grains are colored according to their crystallographic orientations relative to ND. Note difference in scales.
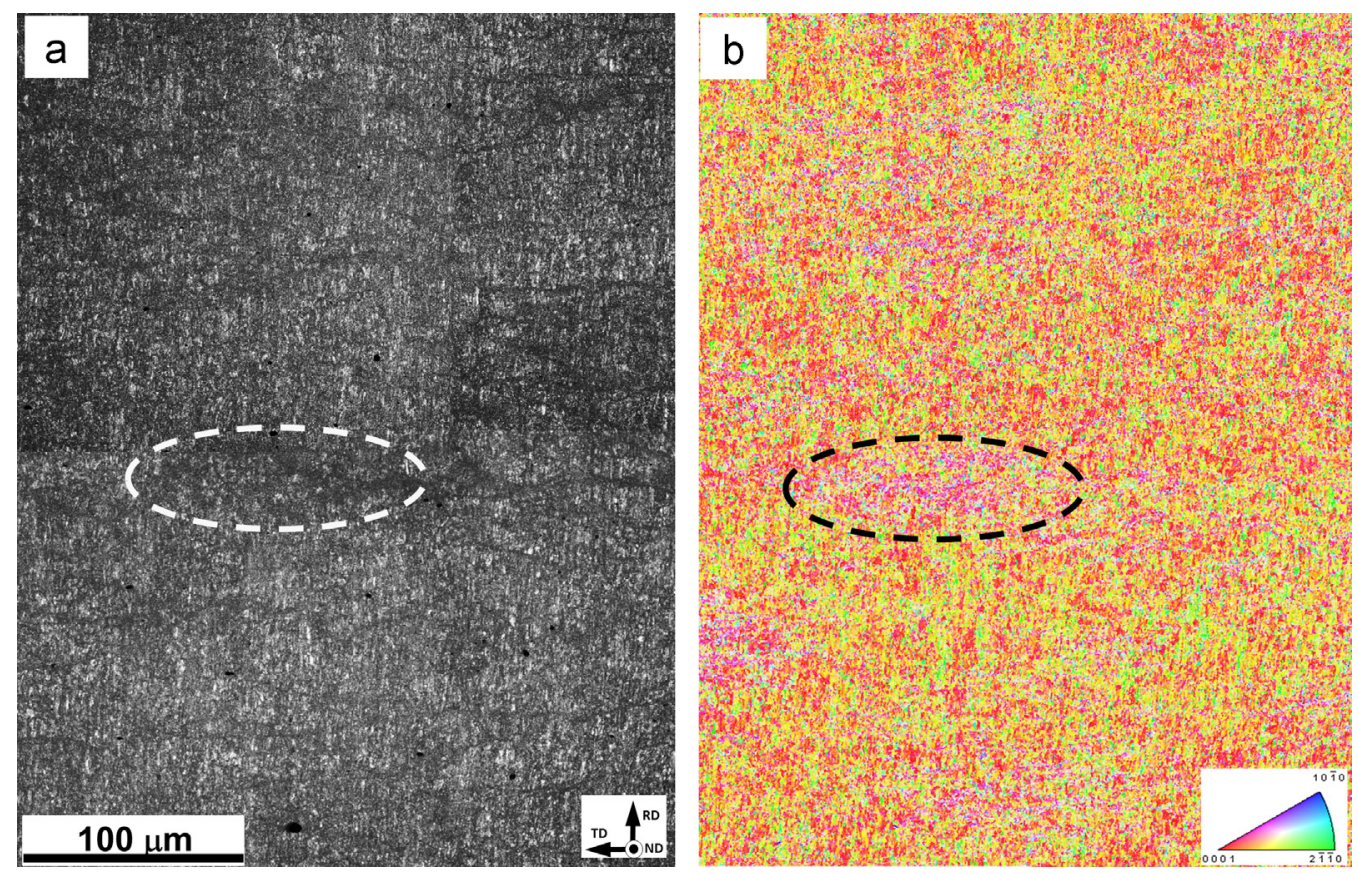

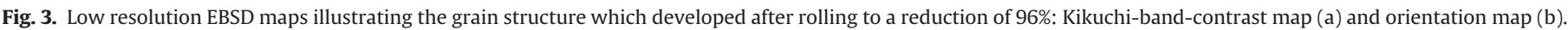

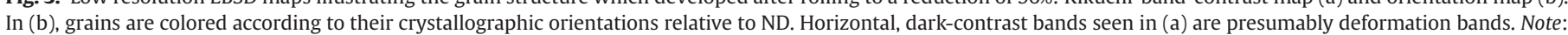

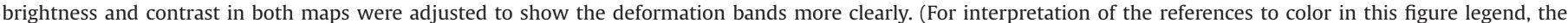
reader is referred to the web version of this article.)

maps in Fig. 4, LABs, HABs, $65^{\circ}\langle 10 \overline{10}\rangle$ and $85^{\circ}\langle 2 \overline{1} \overline{10}\rangle$ boundaries are depicted with red, black, blue and green lines, respectively. In alpha-titanium, the two latter boundary types often outline compression $\{11 \overline{2} 2\}\langle\overline{1} \overline{1} 23\rangle$ twins and tensile $\{10 \overline{1} 2\}\langle\overline{1} 011\rangle$ twins, respectively. The effect of rolling strain on grain size is given in
Fig. 5. With the exception of the unrolled material, grain size statistics were derived from the high-resolution EBSD maps obtained with a scan step size of $0.1 \mu \mathrm{m}$ (Table 2). Three different regimes of microstructure evolution were noted pertaining to low, intermediate, and large deformations. 

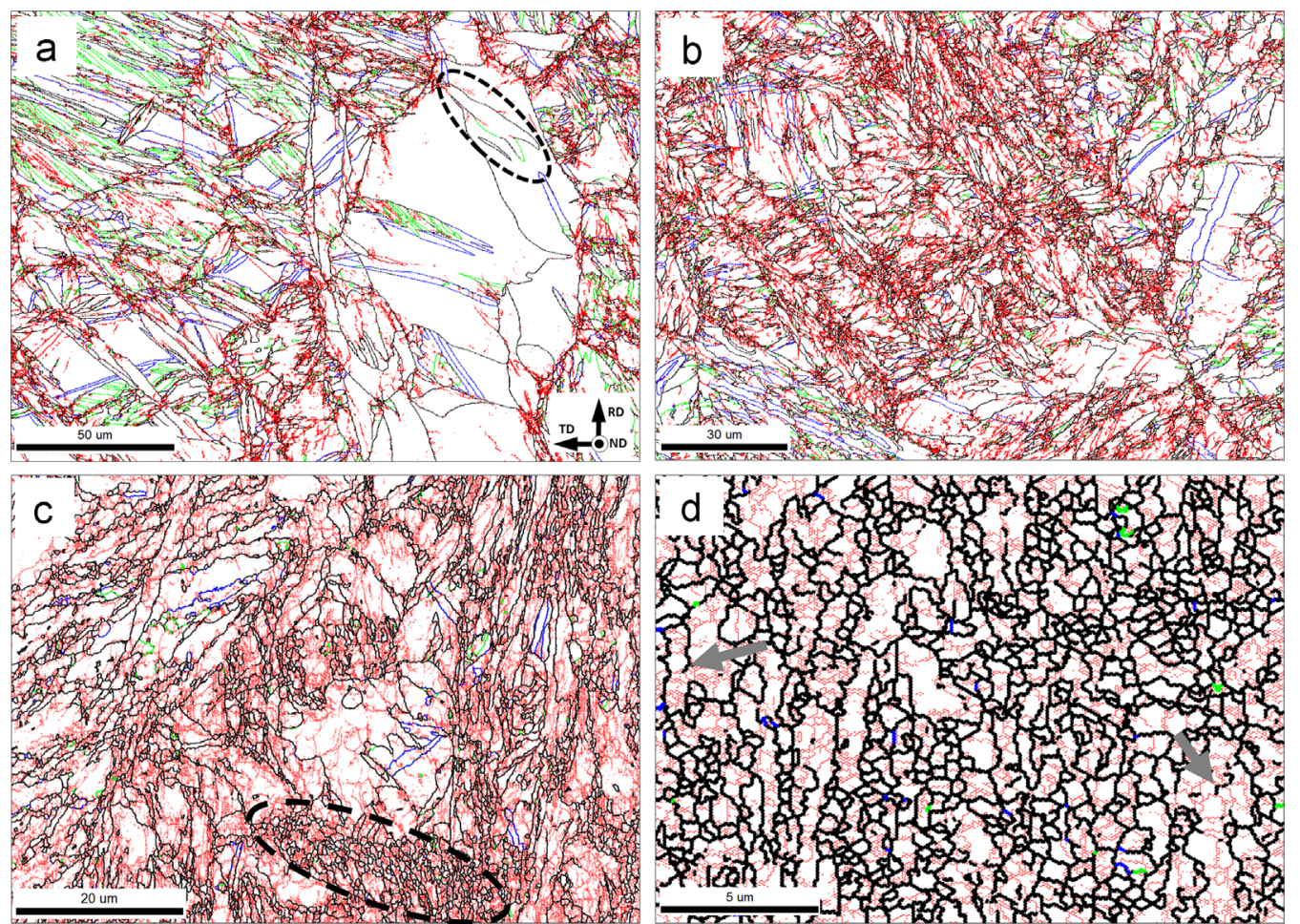

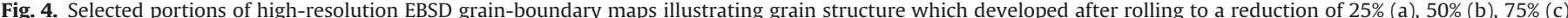

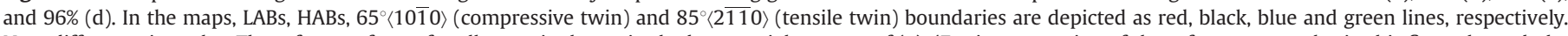

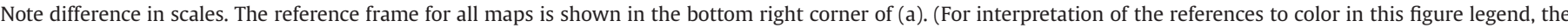
reader is referred to the web version of this article.)

a

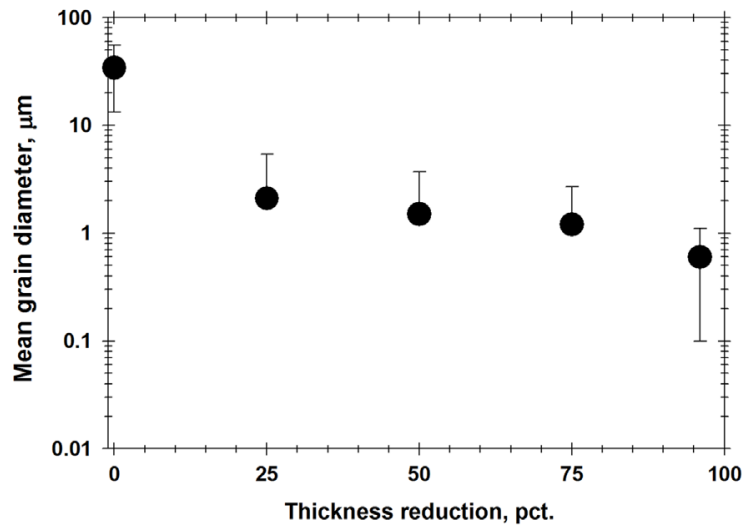

b

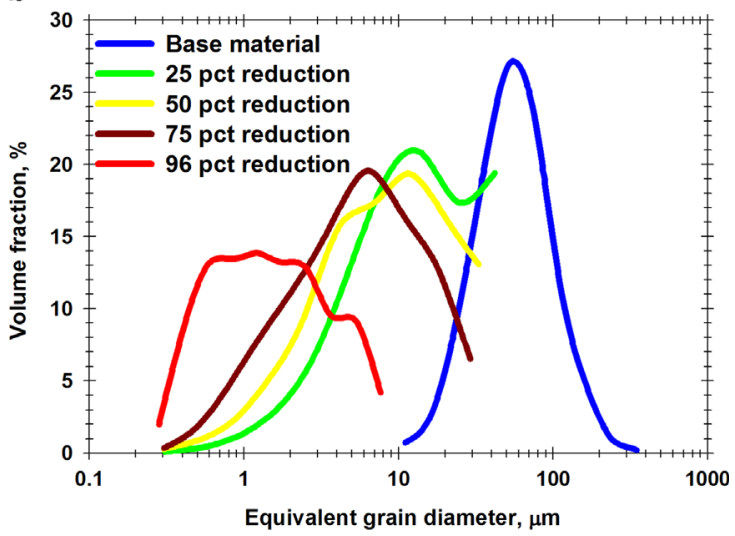

Fig. 5. Effect of rolling reduction on mean grain size (a) and grain-size distribution (b). Error bars in (a) indicate the standard deviation.

\subsubsection{Low strains}

As expected, the microstructures in samples rolled to $25 \%$ and $50 \%$ thickness reduction were dominated by mechanical twinning (Figs. 2a, b and 4a, b). In agreement with prior work [e.g., 4,6], some of the grains were untwinned (or only slightly twinned) thus creating a characteristic microstructural inhomogeneity (Fig. 2a and $\mathrm{b}$ ). The misorientation across the twin boundaries was typically close to $65^{\circ}\langle 10 \overline{1} 0\rangle$ or $85^{\circ}\langle 2 \overline{1} \overline{1} 0\rangle$ (Fig. $4 \mathrm{a}$ and b), thus indicating the activation of $\{11 \overline{2} 2\}\langle 11 \overline{2} \overline{3}\rangle$ compressive and $\{10 \overline{1} 2\}\langle 10 \overline{1} \overline{1}\rangle$ tensile twins, respectively. Surprisingly, the tensile twins were often observed within compression twins (Fig. 4a) thus presumably originating from secondary twinning [14,15]. Moreover, the misorientation of the twin boundaries was found to deviate frequently from the exact twin-matrix relationship with some of the boundary segments transforming into random (nontwin) HABs; an example of an almost completely transformed twin is circled in Fig. 4a. As described in the literature, this phenomenon is typically attributed to strain-induced crystallographic rotation of the twin and matrix from their initial orientations $[8,16]$.

LABs were typically found to cluster in (or near) the twinned regions (Fig. 4a and b). This effect was presumably associated with relatively easy slip within twinned regions $[13,17,18]$. The LABs were usually short, curved, and irregular in appearance. The specific LAB morphology suggested relatively complex slip character in the vicinity of the twins, and may be surmised to be mandated primarily by the maintenance of strain compatibility at twin/matrix interfaces.

The mean grain size was greatly reduced to $\sim 2 \mu \mathrm{m}$ (Fig. 5a) due to twinning. The grain-size distributions were relatively broad (Fig. 5b), thus reflecting the inhomogeneous character of the microstructure. 


\subsubsection{Intermediate strains}

After rolling to a $75 \%$ thickness reduction, remnants of the original grains disappeared almost completely from the microstructure (Fig. 2c). In agreement with findings in the literature [3$10,17,18]$, twinning seemed to be suppressed at this level of reduction. In addition, the twin boundaries were almost completely transformed into HABs with misorientations different from those associated with twinning (Fig. 4c). In the relatively coarse, untwinned regions, LABs with relatively low misorientations were arranged as less-highly developed subgrain structures (Fig. 4c). In contrast, boundaries in the twinned areas often acquired misorientations over $15^{\circ}$ thus transforming them into clusters of fine grains (e.g., area circled in Fig. 4c). This shifted the peak of the grain-size distribution toward smaller sizes (Fig. 5b). Nevertheless, the overall global grain refinement was rather small (Fig. 5a) due to the heterogeneity of the microstructure.

\subsubsection{Large strains}

The distinctive feature of the macroscale structure of material rolled to a $96 \%$ thickness reduction was the formation of deformation bands. In Kikuchi-band-contrast maps, these features appeared as dark bands oriented nearly perpendicular to the RD (Fig. 3a). The deformation bands had a somewhat specific crystallographic orientation (Fig. 3a and b); this behavior is discussed in more detail in Section 4.2.1. The thickness of individual bands was found to be comparable to the grain size. However, several bands often clustered together thereby producing coarser-scale features (selected areas in Fig. 3a and b). The deformation bands were also noted by optical microscopy (supplementary materials, Fig. S2). Deformation banding in heavily rolled titanium is often reported in the literature $[3,19]$. It has been suggested that this process facilitates strain accommodation at large rolling strains (thus replacing twinning) [4] and may significantly refine grain structure [3].

The microstructure after $96 \%$ thickness reduction was also significantly changed. Most of the coarse remnants of the original grains seen at lower strains had disappeared at this level of deformation (Fig. 3b). This behavior narrowed the grain-size distribution somewhat (Fig. 5b), and the microstructure thus became more homogeneous. The evolved microstructure consisted of irregularly shaped, coarser $(\sim 5 \mu \mathrm{m})$ grains elongated in the RD

a
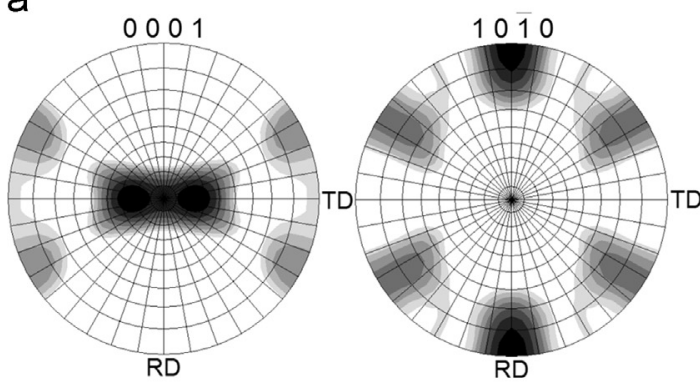

C

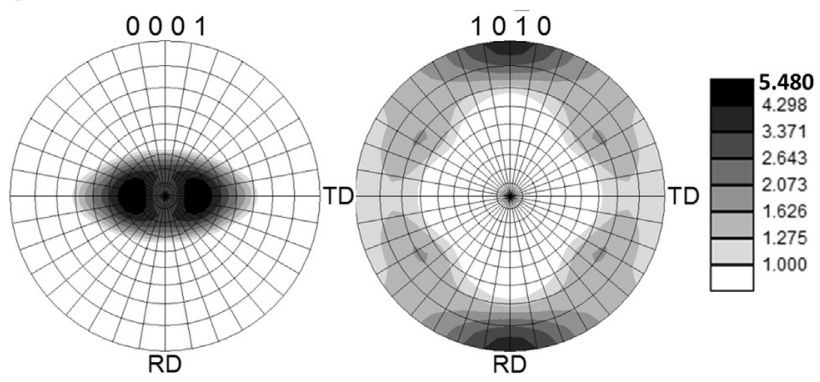

intermixed with fine $(<0.5 \mu \mathrm{m}$ ) equiaxed grains (Fig. 4d). Furthermore, the circle-equivalent mean grain size was reduced twofold to $\sim 0.6 \mu \mathrm{m}$ (Fig. 5a) relative to the structure after $75 \%$ reduction; the mean grain size measured by the intercept method was even smaller, i.e., $\sim 0.3 \mu \mathrm{m}$ in the RD and $0.25 \mu \mathrm{m}$ in the TD.

The grains contained some LAB substructure but almost no twins (Fig. 4d). The sub-boundaries often included HAB segments; several examples are shown by arrows in Fig. 4d. These observations suggested that microstructure development at high strains was influenced by a LAB-to-HAB-transformation process.

\subsection{Texture}

Texture formation was quantified with (0002) and (1010) pole figures (Fig. 6) derived from low-resolution EBSD maps.

After 25\% reduction, the texture was dominated by what appeared to be a 'transient' $\{0002\}\langle 10 \overline{1} 0\rangle$ component (Fig. 6a). The formation of this component is described in the literature as being due to the activation of multiple twins $[6,20]$. For rolling reductions of $50 \%$ and $75 \%$, on the other hand, a split-basal texture, $\{0002\}\langle 10 \overline{1} 0\rangle \pm 30^{\circ}$ toward the TD had evolved (Fig. $6 \mathrm{~b}$ and c). This is also an expected result [6-10]; this texture is believed to be stable during rolling and originates from a balance between twinning, prism slip, and $\langle c+a\rangle$ slip [9]. Formation of this texture is thought to contribute (at least partially) to the rapid drop of the strain hardening rate observed in this strain range (Fig. 1b).

After the 96\% reduction, the split-texture components sharpened substantially (Fig. 6d) and shifted slightly to produce $\{0002\}\langle 10 \overline{1} 0\rangle$ $\pm 40^{\circ}$ toward the TD. In addition, the orientation spread around the major split component increased toward the TD (Fig. 6d). This effect in heavily rolled titanium has also been reported previously [5,6,21]. Although its origin is not completely clear, it is believed it may be related with prism and pyramidal slip [22].

\subsection{Misorientation distribution}

Misorientation data derived from the high-resolution EBSD maps were quantified in terms of the misorientation-angle and misorientation-axis distributions (Figs. 7a and 8, respectively). The misorientation angle data were characterized in terms of specific b

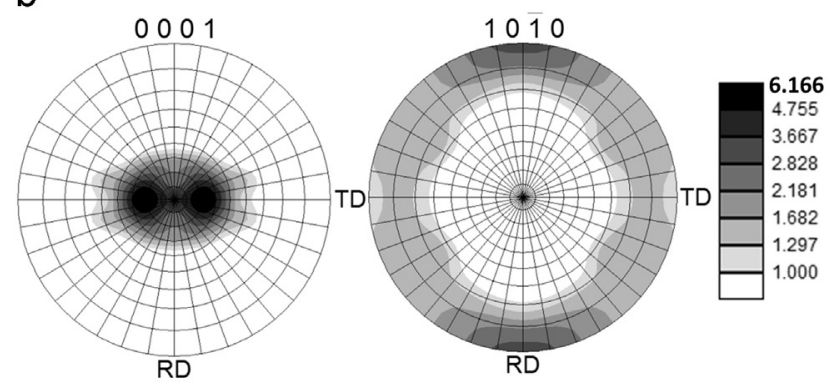

d

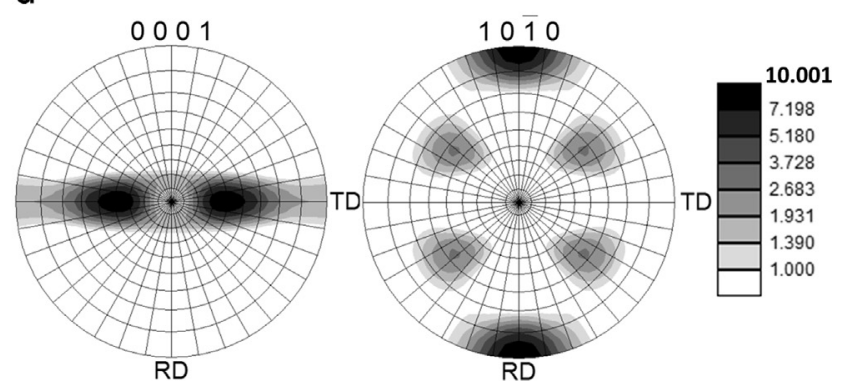

Fig. 6. (0002) and (10̄̄0) pole figures showing the texture developed after rolling reductions of $25 \%$ (a), $50 \%$ (b), $75 \%$ (c), and $95 \%$ (d). 


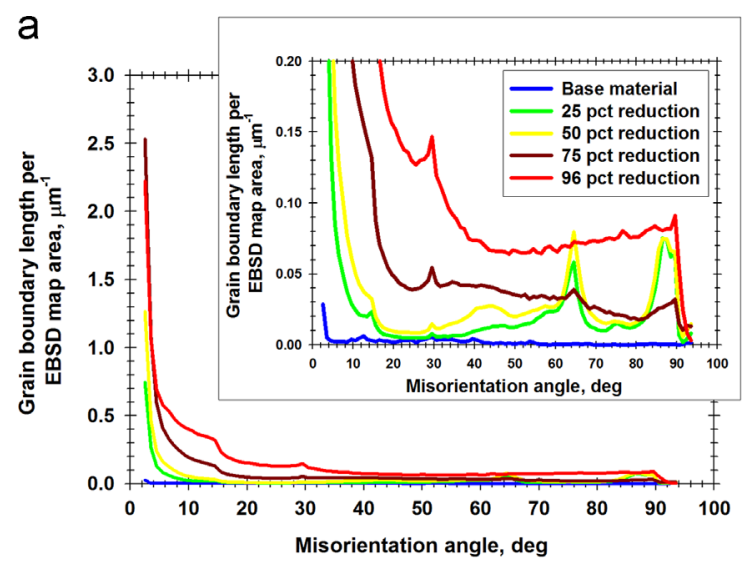

b
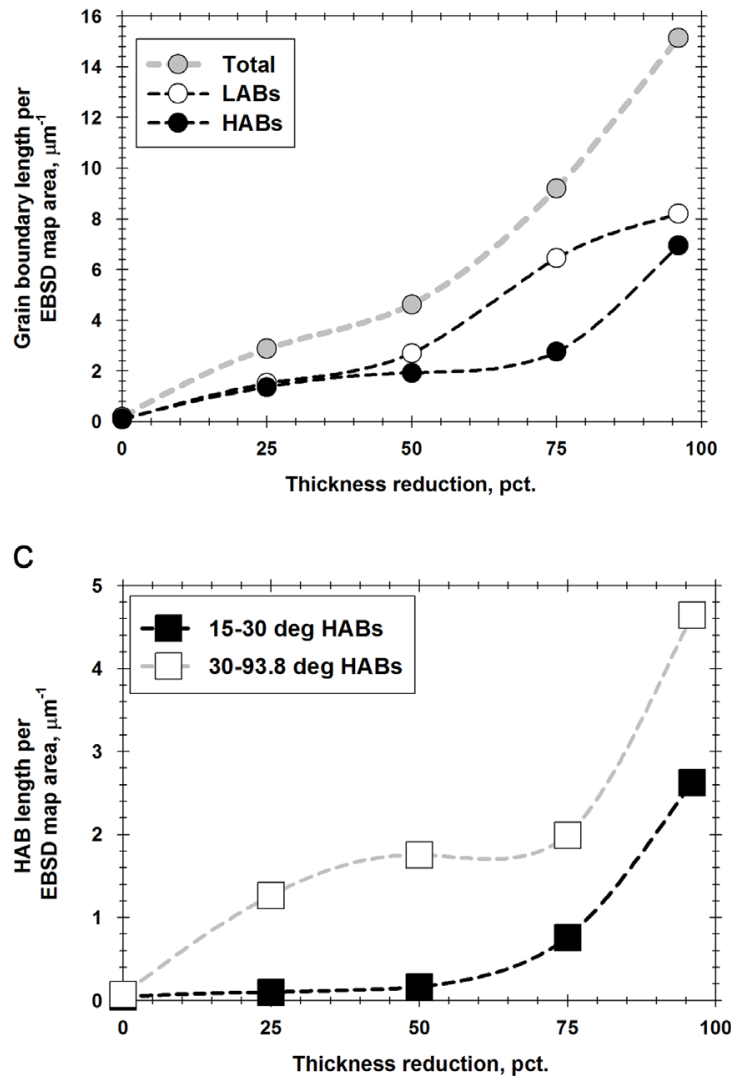

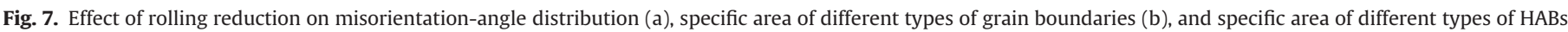
(c). In (a), the insert shows the high-angle region of the distribution in more detail.

grain-boundary area, i.e., the total grain-boundary length for a given misorientation angle (or small range of misorientation angles) divided by the area of the EBSD map. This metrics provides a direct comparison of grain-boundary characteristics for different rolling reductions, thus enabling more reliable determination of the key physical mechanisms governing microstructure evolution. The effect of rolling strain on specific area of different types of grain boundaries is shown in Fig. 7b.

\subsubsection{Dislocation boundaries originating from prism slip}

The rotation axes of (low-angle) dislocation boundaries impose a constraint upon the slip modes which are potentially active during deformation and thus may convey useful information about slip activity per se. It is generally accepted that the slip activity of the $p$ slip system rotates the crystalline lattice around the axis $\bar{w}_{p} \|(\bar{b} \times \bar{n})_{p}$, where $\bar{b}$ is Burger's vector and $\bar{n}$ is the slip plane normal. To maintain strain compatibility requirements, however, several slip systems (or even different deformation modes) usually operate within a grain and thus contribute to the formation of a dislocation boundary. Therefore, the misorientation axis of the boundary depends on the contribution of each slip system (mode) and thus is difficult to predict.

Considering the significance of prism slip in titanium, however, it can be instructive to examine those dislocation boundaries possibly originating from this slip mode. Of particular interest is the fact that all prism slip systems provide a rotation around the same [0001] axis. In hexagonal close-packed crystals, the [0001] direction is a six-fold rotation axis. On the other hand, the (0002) basal plane is a plane of mirror symmetry. Thus the maximum rotation angle about [0001] is $30^{\circ}$. This simple idea agrees well with the measured misorientation-axis distributions shown in Fig. 8; i.e., it is apparent that the region near the [0001] pole 'empties' as the misorientation angle approaches and exceeds $30^{\circ}$. Thus, it is important to note that the maximum misorientation across the dislocation boundaries originating from purely prism slip cannot exceed $30^{\circ}$.

It seems useful therefore to broadly categorize the deformationinduced HABs in titanium into two groups: (i) those with misorientations below $30^{\circ}$, (which appear to originate mainly from prism slip) and (ii) those with misorientations above $30^{\circ}$ which tend to be related to deformation twinning as well as possibly other slip modes. The effect of rolling reduction on the specific area of the two types of HABs is shown in Fig. 7c.

\subsubsection{Low strains}

Rolling to $25 \%$ and $50 \%$ thickness reductions gave rise to a significant increase of $\mathrm{LAB}$ area relative to the undeformed condition (Fig. 7a and b). The misorientation axes of the LABs were not randomly oriented but tended to align with the [0001] axis (Fig. 8a and $b$ ). In material rolled to a $25 \%$ reduction, a slight clustering of LAB misorientation axes near the [2 $\overline{1} \overline{1} 0]$ direction was also found (Fig. 8a). After 50\% reduction, however, the LAB cluster near the [21 10$]$ seemed to disappear (Fig. 8b).

In addition to the pronounced development of LABs, the rolled material was characterized by a significant increase in the specific area of $65^{\circ}\langle 10 \overline{1} 0\rangle, 85^{\circ}\langle 2 \overline{1} \overline{1} 0\rangle$, and $\sim 45^{\circ}\langle 5 \overline{1} \overline{4} 3\rangle$ boundaries (Figs. $7 \mathrm{a}$ and $8 \mathrm{a}, \mathrm{b})$. The crystallographic preference of the first two misorientations can be ascribed to $\{11 \overline{2} 2\}$ and $\{10 \overline{1} 2\}$ twins, whereas the latter boundaries could be attributable to 'double' twinning (i.e., the formation of tensile twins inside the compressive twins) [23]. 
a
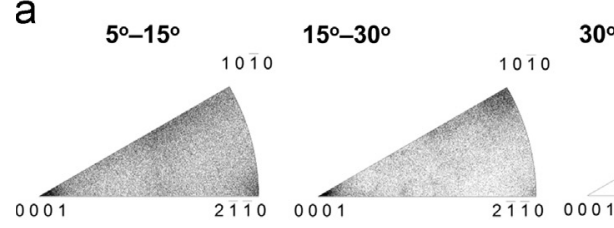

$70^{\circ}-94^{\circ}$

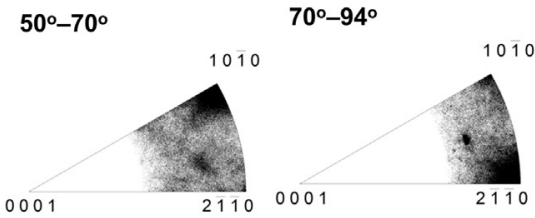

C

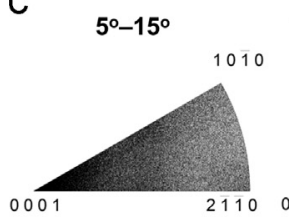

$15^{\circ}-30^{\circ}$

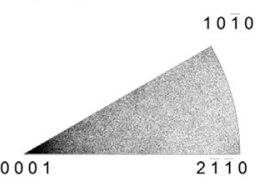

$30^{\circ}-50^{\circ}$

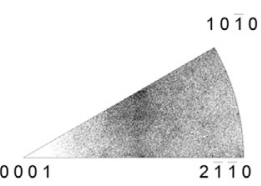

$70^{\circ}-94^{\circ}$
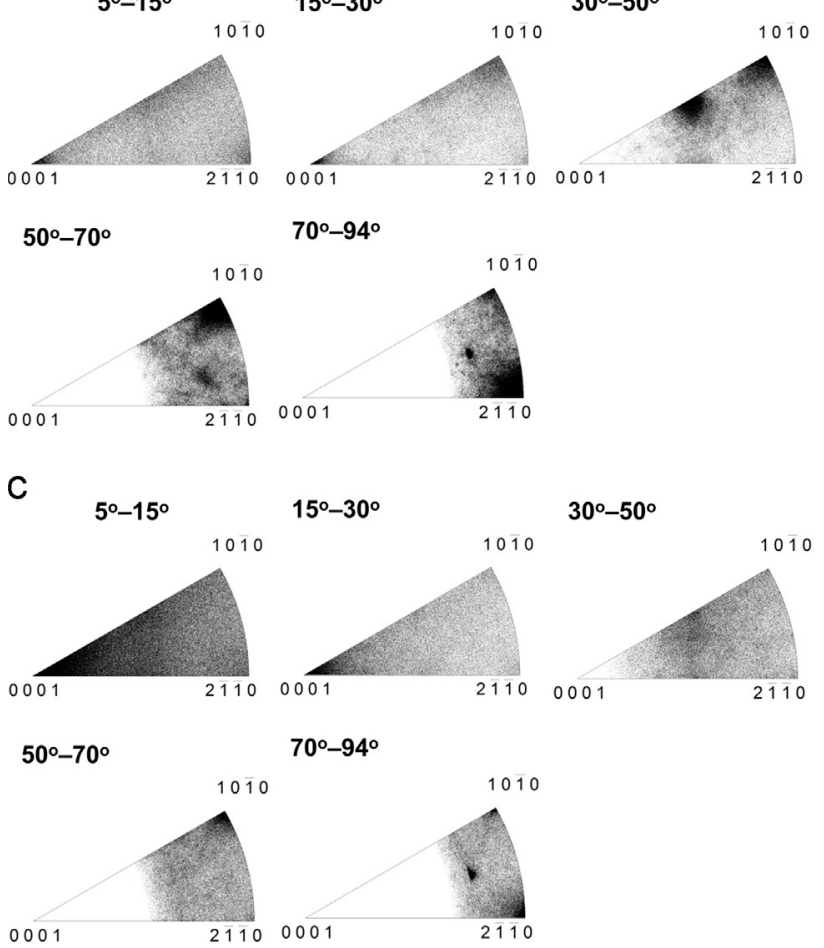

b

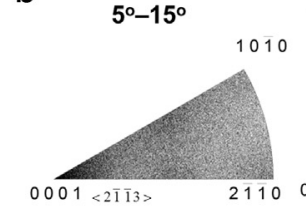

$15^{\circ}-30^{\circ}$

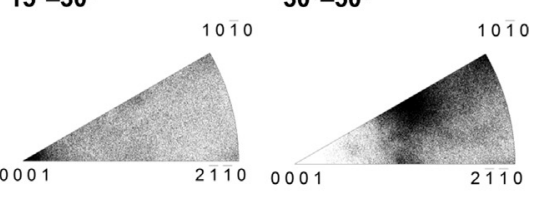

$50^{\circ}-70^{\circ}$

$70^{\circ}-94^{\circ}$

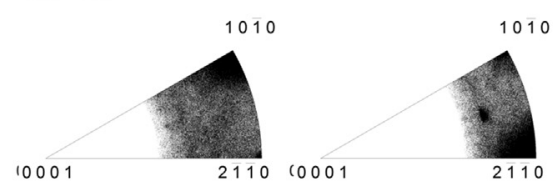

d

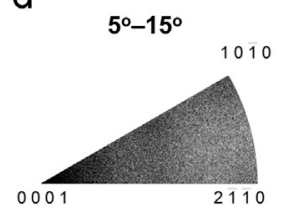

$15^{\circ}-30^{\circ}$
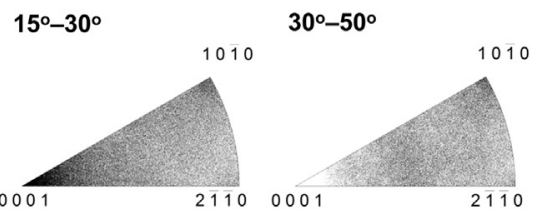

$70^{\circ}-94^{\circ}$

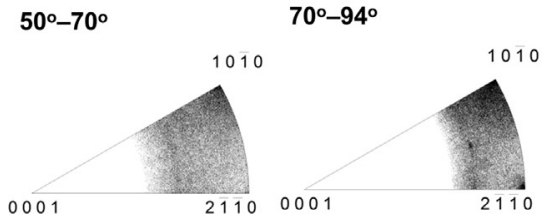

Fig. 8. Misorientation-axis distributions measured in the material rolled to reductions of $25 \%$ (a), $50 \%$ (b), $75 \%$ (c), and $95 \%$ (d).

A slightly increased proportion of $35^{\circ}\langle 10 \overline{1} 0\rangle$ boundaries (associated with $\{11 \overline{2} 1\}\langle\overline{1} \overline{1} 26\rangle$ tension twins) was also found.

The twin-induced peaks in misorientation-angle (Fig. 7a) and misorientation-axis (Fig. 8a, b) distributions were characterized by a substantial spread exceeding normal EBSD errors $\left(\sim 2^{\circ}\right)$. This effect was likely related to the strain-induced transformation of twin boundaries, as discussed in Section 3.2.1.

The significant increase of the HAB area during this level of reduction was almost solely related to boundaries having misorientations above $30^{\circ}$ (Fig. 7c). This confirms that considerable grain refinement observed in this strain range (Fig. 5a) was attributable to deformation twinning.

\subsubsection{Intermediate strains}

After rolling to a $75 \%$ thickness reduction, the LAB area was significantly increased (Fig. 7a and b). This agrees with Ref. [5] reporting extensive formation of deformation-induced boundaries at the rolling strains exceeding 50-60\% The LAB cluster near [0001] was strengthened substantially (Fig. 8c).

The low-angle peak in the misorientation-angle distribution shifted toward high-angle misorientations (Fig. 7a). This presumably reflected a gradual accumulation of misorientations by LABs and subsequent LAB-to-HAB transformation.

Twin-induced peaks were significantly reduced in size (Figs. 7a and $8 \mathrm{c}$ ). This effect can be explained by the progressive straininduced transformation of twins. On the other hand, the specific area of $\mathrm{HABs}$ with misorientation above $30^{\circ}$ did not change notably (Fig. 7c). This perhaps evidences suppression of twinning at large rolling strains and agrees well with findings in the literature $[3-6,9,10]$.

The specific area of HABs with misorientation below $30^{\circ}$ increased considerably during rolling to $75 \%$ reduction (Fig. 7c). A subtle peak near $30^{\circ}$ appeared in the misorientation-angle distribution (Fig. 7a); misorientation axes of the respective boundaries were clustered near the [0001] pole (Fig. 8c).

\subsubsection{Large strains}

In material rolled to the $96 \%$ thickness reduction, the LAB area increased further (Fig. 7b). Specifically, the area of $5-15^{\circ}$ boundaries increased at the expense of $2-3^{\circ}$ boundaries (Fig. 7a), thus implying a pronounced accumulation of misorientation by the LABs. The misorientation-axis distribution for LABs was not noticeably altered in comparison to the observations for the sample rolled to a $75 \%$ reduction (Fig. $8 \mathrm{c}$ versus $\mathrm{d}$ ).

The misorientation-angle distribution showed no clear twininduced peaks (Fig. 7a) at the highest level of reduction. The total twin boundary fraction was found to be only $\sim 1.6 \%$ It was therefore concluded that twinning was negligible, and the boundaries of the former twins were almost completely transformed into non-special HABs.

One of the most striking results for $96 \%$ reduced material was the large increase in the HAB area (Fig. 7b). Although the degree of grain refinement in this strain range appeared to be relatively small (Fig. 5a), the HAB area was almost tripled (Fig. 7b). Of particular interest was the observation that the HAB area enlargement was significantly contributed by HABs with a misorientation above $30^{\circ}$ (Fig. 7c). As suggested in Section 3.4.1, these boundaries could not have originated from purely prism slip.

\section{Discussion}

\subsection{Low and intermediate strains}

Microstructure evolution at relatively low and intermediate strains ( $\leq 75 \%$ ) was found to be in good agreement with prior observations [3-10]. At low strains, microstructure evolution was dominated by the development of $\{11 \overline{2} 2\}\langle 11 \overline{2} \overline{3}\rangle$ compressive and $\{10 \overline{1} 2\}\langle 10 \overline{1} \overline{1}\rangle$ tensile twins which rapidly refined the grain size. The extensive twinning produced a transient basal-texture component.

As the rolling reduction increased, the misorientation across the twin boundaries gradually deviated from the precise twin-matrix 
orientation relationship and the twinned regions were transformed into ordinary grains. Subsequent substructure development within the twinned regions converted them into small equiaxed grains. The progressive grain refinement suppressed twinning and the resultant slip-dominated deformation led to the formation of a split-basal $\{0002\}\langle 10 \overline{1} 0\rangle \pm 30^{\circ}$ TD texture.

It is worth noting as well that LABs tended to preferentially localize in (or near) twinned regions, which resulted in an irregular morphology with their misorientation axes close to [0001].

\subsection{Large strains}

For rolling reductions in the range of $75-96 \%$, significant grain refinement took place, which resulted in the formation of an ultrafine grain structure. Possible explanations for this effect are examined below.

\subsubsection{Deformation banding}

One possible source of the observed grain refinement in heavily rolled material was deformation banding $[3,19]$. The typical appearance of deformation bands in a Kikuchi-band-contrast EBSD map and an orientation EBSD map is shown in Fig. 9a and b, respectively; in both cases, the deformation band is indicated by arrows. The crystallographic orientations within the deformation band as well as the surrounding matrix are given in Fig. 9c and d, respectively. Pole figures revealed that the orientations of the band and the matrix were relatively close. The misorientation between them was estimated as comprising a $\sim 5-10^{\circ}$ rotation around an axis nearly parallel to the TD (Fig. 9c and d). The proximity of the orientations also followed from the EBSD orientation map in Fig. 9b; here, it is seen that the deformation band is difficult to distinguish from the matrix. Because the grain tolerance angle was taken to be $15^{\circ}$, it seems unlikely that deformation banding per se contributed significantly to grain refinement. Moreover, direct grain size measurements showed only a small difference between the bands and the matrix (supplementary materials, Fig. S3.).

Fig. 9c and d also indicated that the deformation bands were not responsible for the large orientation spread around typical split-basal texture seen in (0002) pole figure for 96\% rolled material (Fig. 6d).

\subsubsection{Correlation between texture and $H A B$ development}

The grain refinement in the heavily rolled titanium obviously resulted from the very large increase in the HAB area (Fig. 7b). In this regard, it is of interest to examine a possible relationship between $\mathrm{HAB}$ development and texture evolution at large strains (Fig. 6d).
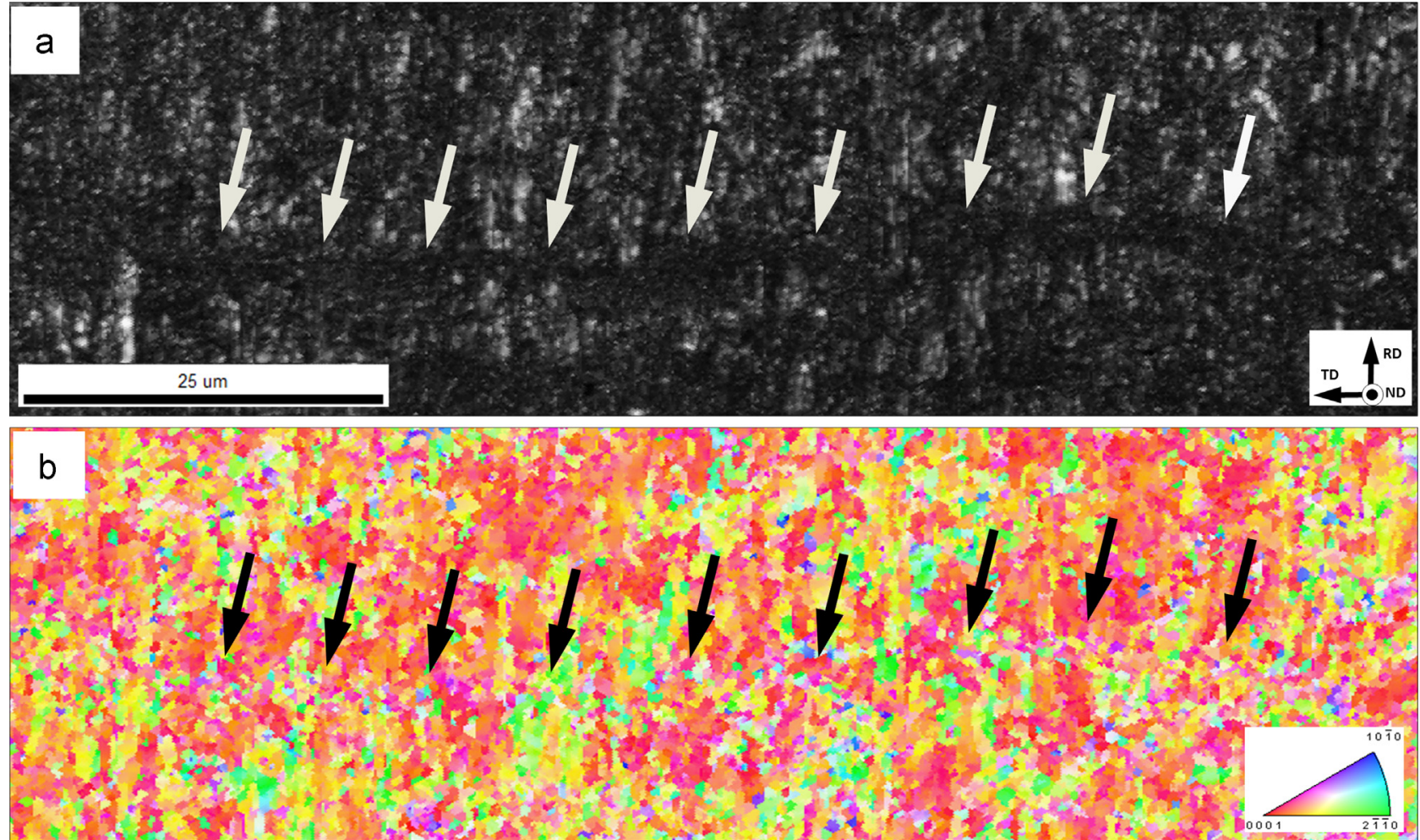

C

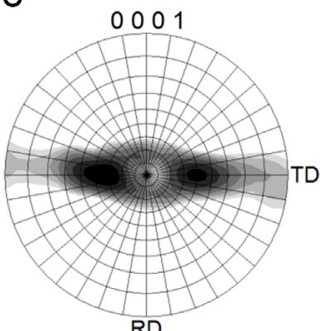

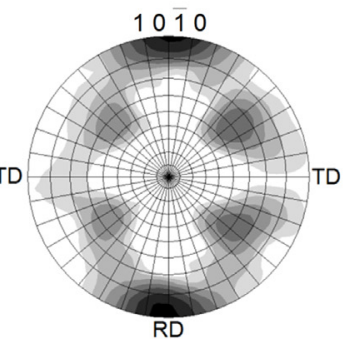

RD d
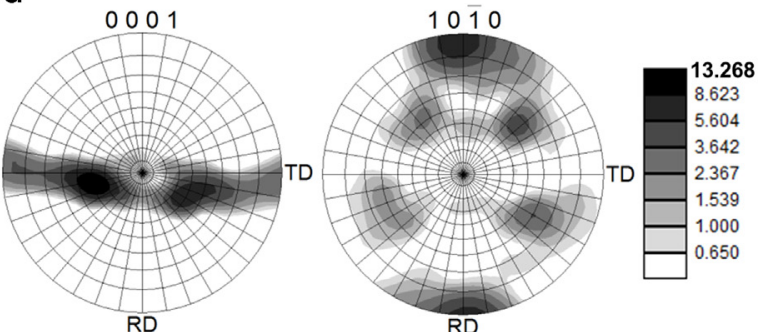

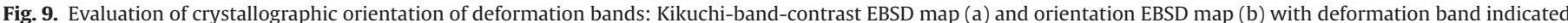
by arrows and (0002) and (1010) pole figures showing crystallographic orientation of the matrix (c) and the deformation band (d). 


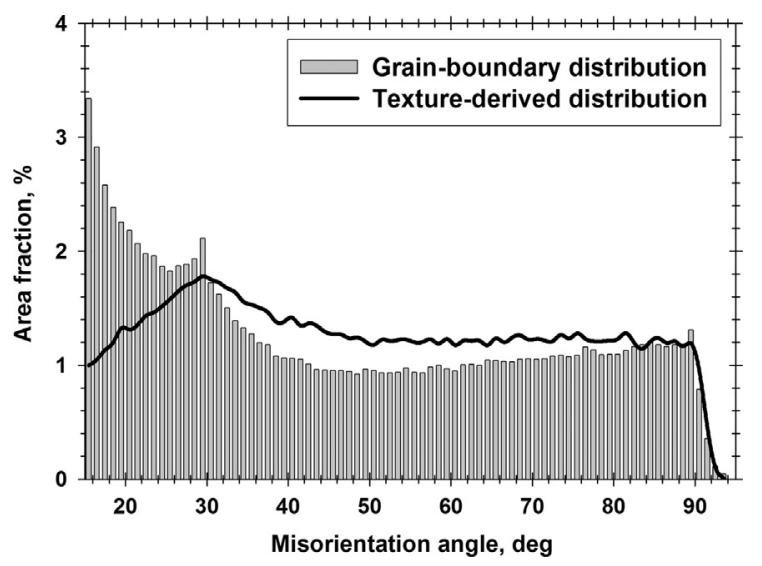

Fig. 10. Comparison of the high-angle portions of the measured (grain-boundary) misorientation distribution and texture-derived distribution for material rolled to a reduction of $96 \%$.

To assess the effect of texture on HAB evolution, misorientation distributions for the high-angle range were derived from the texture data and compared with the grain-boundary misorientation distribution in Fig. 10. In contrast to the measured misorientation distribution (based on data for neighboring pixels), the texture-derived distribution was calculated assuming no spatial correlation between pixels. In other words, all possible misorientations between the sampled pixels (including noncontiguous ones) were calculated. Fig. 10 demonstrates that the measured distribution shows approximate agreement with the texturederived one in the misorientation range above $30^{\circ}$. This suggests that the development of these boundaries was closely linked with the texture change.

It seems therefore that the extensive HAB formation (and related grain refinement) was associated with the appearance of the specific orientation spread near typical split-basal texture (Fig. 6d). In this context, the reason for this effect becomes of particular interest.

\subsubsection{Possible activation of $\langle c+a\rangle$ slip}

As discussed in the previous section, the reason for the appearance of orientation spread of the split-basal components at very high reductions is of particular interest. In principle, texture evolution in heavily rolled material may be influenced by the partial relaxation of constraint imposed by adjacent grains due to grain flattening and a related reduction in the number of grain neighbors [24]. However, due to extensive twinning at low strains, the grains in heavily rolled titanium were found to be irregularly shaped and the number of the grain neighbors appeared to be rather high (Fig. 4c and d). Accordingly, strain compatibility requirements seem to be fairly high over the entire range of reductions investigated.

Another possible reason for the texture change in heavily rolled titanium (Fig. 6d) may be activation (or enhancement) of different slip (or twinning) modes. It is worthwhile therefore to consider this idea in terms of specific microstructure and texture developed in material reduced in thickness by $96 \%$. First, basal slip is believed to shift texture peaks toward the ND [9]. Hence, it is unlikely that the observed smearing toward the TD was associated with basal slip. The observed texture change also could not be attributed to twinning because this mechanism was likely suppressed at high strains as discussed in Section 3.4.4. Furthermore, it was surmised that prism slip could not provide the abundant misorientations exceeding $30^{\circ}$ developed at large strains (Fig. 7c).
In view of the above considerations, the possible enhancement of pyramidal slip deserves particular attention. Because twinning was suppressed after $\sim 50 \%$ thickness reduction, strain accommodation along the [0001] direction in hexagonal close-packed metals may be achieved only via $\langle c+a\rangle$ pyramidal slip. In titanium, this type of slip is believed to shift the texture peaks in the (0002) pole figure toward the TD [22], as observed in the present measurements (Fig. 6d). The enhancement of the $\langle c+a\rangle$ slip should rapidly increase dislocation density and thus intensify the process of grain-boundary development. This also agrees well with experimental observations (Fig. $7 \mathrm{~b}$ and $\mathrm{c}$ ). Although $\langle\mathrm{c}+\mathrm{a}\rangle$ slip is characterized by a relatively high critical resolved shear stress, significant material strengthening (i.e., hardening of the prism $\langle a\rangle$ and basal $\langle a\rangle$ systems during heavy reductions) (Fig. 1a) could have contributed to its activation. It is thus concluded that the observed texture change and related enhancement of grain refinement originated from the activation of pyramidal slip.

\section{Conclusions}

Grain structure development during cold rolling of commercial-purity titanium was examined with particular attention to the behavior at large strains (i.e., reductions $>75 \%$ ). The main conclusions from this work are as follows:

1. At low thickness reductions ( $\leq 75 \%$ ), microstructure is greatly refined due to twinning. Despite extensive substructure development in the twinned regions (which gradually transform the twins into fine-grained structure), the mean grain size for reductions of $25-75 \%$ does not change significantly. The suppression of twinning at $\sim 50 \%$ reduction and the predominance of slip give rise to a conventional 'split-basal' $\{0002\}\langle 10 \overline{1} 0\rangle \pm 30^{\circ}$ TD texture.

2. Larger ( $>75 \%$ ) deformations lead to significant microstructure and texture changes. Extensive deformation banding is found. The HAB area almost triples, thus producing an ultrafinegrained structure with a mean grain size of $0.6 \mu \mathrm{m}$. Additionally, the orientation spread around the typical 'split-basal' rolling texture substantially increased.

3. Deformation bands formed at high reductions are not highly misoriented relative to the surrounding matrix. Thus, it is unlikely that they provide a significant contribution to grainstructure evolution. Rather, the extensive grain refinement and texture changes at large strains are likely attributable to the activation of $\langle\mathrm{c}+\mathrm{a}\rangle$ slip.

\section{Appendix A. Supplementary materials}

Supplementary data associated with this article can be found in the online version at http://dx.doi.org/10.1016/j.msea.2014.03.141.

\section{References}

[1] R.Z. Valiev, R.K. Islamgaliev, I.A. Alexandrov, Prog. Mater. Sci. 45 (2000) $103-189$.

[2] V.V. Stolyarov, L. Zeipper, B. Mingler, M. Zehetbauer, Mater. Sci. Eng. A 476 (2008) 98-105.

[3] D. Terada, S. Inoue, N. Tsuji, J. Mater. Sci. 42 (2007) 1673-1681.

[4] Y.B. Chun, S.H. Yu, S.L. Semiatin, S.K. Hwang, Mater. Sci. Eng. A 398 (2005) 209-219.

[5] S.V. Zherebtsov, G.S. Dyakonov, A.A. Salem, S.P. Malysheva, G.A. Salishchev, S.L. Semiatin, Mater. Sci. Eng. A 528 (2011) 3474-3479.

[6] N. Bozzolo, N. Dewobroto, H.R. Wenk, F. Wagner, J. Mater. Sci. 42 (2007) 2405-2416.

[7] S.V. Zherebtsov, G.S. Dyakonov, A.A. Salem, V.I. Sokolenko, G.A. Salishchev, S.L. Semiatin, Acta Mater. 61 (2013) 1167-1178.

[8] E.V. Nesterova, V.V. Rybin, Phys. Met. Metall. 59 (1985) 169-179. 
[9] H.P. Lee, C. Esling, H.J. Bunge, Textures Microstruct. 7 (1988) 317-337.

[10] S. Nourbakhsh, T.D. O'Brien, Mater. Sci. Eng. 100 (1988) 109-114.

[11] D.P. Field, Ultramicroscopy 67 (1997) 1-9.

[12] F.J. Humphreys, J. Microscopy 195 (1999) 170-185.

[13] A.A. Salem, S.R. Kalidindi, R.D. Doherty, Acta Mater. 51 (2003) 4225-4237.

[14] I.J. Beyerlein, J. Wang, M.R. Barnett, C.N. Tome, Proc. R. Soc. A 468 (2012) 1496-1520.

[15] H.E. Kadiri, J. Kapil, A.L. Oppedal, L.G. Hector Jr., S.R. Agnew, M. Cherkaoui, S.C. Vogel, Acta Mater. 61 (2013) 3549-3563.

[16] G. Salishchev, S. Mironov, S. Zherebtsov, A. Belyakov, Mater. Character. 61 (2010) 732-739.

[17] A.A. Salem, S.R. Kalidindi, R.D. Doherty, S.L. Semiatin, Metall. Mater. Trans. 37A (2006) 259-268
[18] A.A. Salem, S.R. Kalidindi, S.L. Semiatin, Acta Mater. 51 (2005) 3495-3502.

19] D.K. Yang, P. Cizek, P.D. Hodgson, C.E. Wen, Acta Mater. 58 (2010) 4536-4548.

[20] Y. Zhong, F. Yin, K. Nagai, J. Mater. Res. 23 (2008) 2954-2966.

[21] M.J. Philippe, M. Serghat, P. Van Houtte, C. Esling, Acta Metall. Mater. 43 (1995 $1619-1630$.

[22] S. Zaefferer, Mater. Sci. Eng. A 344 (2003) 20-30.

[23] N. Bozzolo, L. Chan, A.D. Rollett, J. Appl. Cryst. 43 (2010) 596-602.

[24] U.F. Kocks, G.R. Canova, Deformation of Polycrystals: Mechanisms and Microstructures, in: N. Hansen, A. Horsewell, T. Leffers, H. Lilholt (Eds.), Riso National Laboratory, Roskilde, 1981, pp. 35-44. 